\title{
La violencia entre pares dentro del ambiente escolar
}

Francisco Loya Loya

abelloya2004@hotmail.com

\section{Resumen}

La violencia que se vive diariamente en la sociedad tanto en México como en otros países, hace necesario que se realicen trabajos de investigación relacionados con este fenómeno, sobre todo en espacios educativos para avanzar en el conocimiento respecto de la influencia de esta problemática en la formación de nuevos ciudadanos. El presente trabajo de investigación tiene como objeto conocer la percepción que tienen los participantes dentro del proceso educativo como son alumnos, docentes y directivos, así como los padres de familia, dentro de los planteles escolares de educación básica en relación con la violencia que se genera dentro de las instituciones educativas, específicamente en la primaria.

\section{Palabras clave}

Ambiente educativo, clima escolar, planteles escolares, problemas estudiantiles, violencia escolar.

\section{Antecedentes}

La violencia entre pares dentro del ambiente escolar es una situación que es necesario enfrentar y frenar en el momento en el que se manifiesta, es preferible no esperar a que esta condición humana rebase los límites y se convierta en una situación caótica y fuera de control.

El logro en la creación de ambientes seguros de aprendizaje es un aspecto ineludible, los esfuerzos de los trabajadores de la educación, especialmente docentes y directivos que laboran en escuelas de educación básica necesitan estar enfocados hacia la búsqueda de espacios de aprendizaje libres de violencia. Desafortunadamente el mundo moderno y la forma de vida tan vertiginosa, presentan aspectos que impiden el desenvolvimiento normal de los alumnos en las instituciones educativas.

El maestro, conjuntamente con la sociedad en general, enfrenta un reto inevitable, requiere buscar estrategias que permitan prevenir, enfrentar y resolver las problemáticas que aquejan a la vida cotidiana dentro de los planteles educativos, como lo es la violencia entre pares.

\section{Objetivos}

Con base a la necesidad de buscar solución a problemáticas cotidianas dentro de los planteles escolares, es importante enfocar la atención hacia el conocimiento de dichas situaciones, por lo tanto este trabajo de investigación se origina a partir de la siguiente interrogante: ¿Cómo perciben los diferentes actores educativos de la escuela primaria la violencia entre pares?

Los objetivos a los que responde la investigación planteada en el presente documento son:

Objetivos Específicos

1. Comparar la percepción sobre violencia entre pares de los diferentes 
actores educativos de la escuela primaria: alumnos, docentes, directivos y padres de familia.
2. Identificar algunos aspectos importantes relacionados con la violencia entre pares.

\section{Fundamentos}

La sociedad atraviesa por un momento crítico en cuanto se refiere la pérdida de valores, acrecentamiento de la pobreza, ausencia de medios de subsistencia y falta de empleo. El mundo globalizado se basa en el dominio económico de algunos países sobre otros, dejando atrás identidades culturales $\mathrm{y}$ formando una nueva cultura en la que se manifiesta una crisis económica, social, política y cultural en las sociedades del mundo.

En América Latina, incluido México, se ha acentuado la inequidad, con las crisis en sus sistemas educativos que sólo buscan la ganancia en términos empresariales y no el bienestar social. Este tipo de gobierno ha traído consecuencias negativas para la vida diaria de las personas, por ejemplo, podemos observar directamente cómo la violencia está acrecentándose de manera acelerada y alarmante. Día a día se observa en los hogares, centros escolares y de trabajo, en la calle o en cualquier sitio indicios de una vida donde desfavorablemente la violencia se hace presente de manera cada vez más común.

Una de las metas educativas de la Organización de Estados Iberoamericanos para el año 2021, propuesta por los ministros de educación de estos países en San Salvador en el año 2008, enfocada a la educación en valores y para la ciudadanía, dice:

Es preciso... fomentar la participación en el ámbito escolar y propiciar un clima satisfactorio que ayude a los alumnos a vivir juntos $\mathrm{y}$ a ser tolerantes y solidarios. Es necesario promover innovaciones y encontrar estrategias que sean atractivas para ellos y les permitan, a través de la acción, aprender el ejercicio de los valores. (OEI, 2010, p. 250

En la actualidad los problemas sociales y económicos se ven recrudecidos en los grupos marginados con la crisis financiera que se vive en algunos países. La UNESCO (2010) pide como estrategia, fortalecer el entorno de aprendizaje. Desafortunadamente esta condición resulta no sólo insuficiente, sino fuera del alcance de la realidad para muchas naciones que se ven rebasadas por la violencia dentro de su esquema social.

Margarita Zorrilla (2002) expresa que a pesar de un entorno desfavorable, el funcionamiento de una escuela influye en el buen nivel de logro académico, formativo y social de los alumnos, con base en el buen liderazgo, metas compartidas entre todos los actores dentro del proceso educativo, propiciando un buen ambiente para el aprendizaje, profesionalizando docentes y sobre todo teniendo altas expectativas sobre los alumnos.

Hoy en día el problema de la violencia entre pares forma parte de la vida cotidiana en la escuela y para estar en condiciones de disminuir la situación es deseable entender el fenómeno.

Carrillo y Prieto señalan que:

La violencia es considerada como una actitud o comportamiento que constituye una violación o un arrebato al ser humano de algo que le es esencial como persona: su integridad física, psíquica, moral; sus derechos y libertades. Puede provenir de personas o instituciones, y puede ser pasiva o activa, ya que, aparte de la violencia directa (golpes, destrucción visible, entre otras), también se encuentran formas indirectas o sutiles, como las psicológicas, tal vez más difíciles de 
reconocer, pero que son cotidianas en ámbitos como la escuela (Carrillo y Prieto en Furlán et al., 2010, p. 71).

En el año 2005, Díaz-Aguado, comenta que existen una multitud de factores por los que un alumno desde temprana edad pueda presentar una conducta violenta, entre éstos está la exclusión social o sentimiento de exclusión, así como la ausencia de límites y la exposición cotidiana a los medios de comunicación. En edades más avanzadas se menciona la influencia de bandas involucradas en violencia e inclusive con la facilidad de conseguir armas. No pasamos por alto la forma en que la sociedad percibe la violencia y la justifica de cierta manera.

Se ha comentado ya que la violencia entre pares es una situación muy preocupante y además tan frecuente a lo largo y ancho del territorio nacional. Así mismo se ha observado cómo crece la tendencia de tener un comportamiento violento por parte de ciertos estudiantes dentro de los planteles escolares, donde las actitudes agresivas se muestran de manera más común. Actualmente algunos alumnos dejan ver una evidente agresividad y falta de respeto hacia los demás compañeros, e inclusive hacia el personal de la escuela. Las instituciones educativas presentan un factor de cambio y de mejoramiento, los docentes y directivos desde su posición, intentan reforzar en el trabajo diario la vivencia de valores que los alumnos presentan desde su formación en el hogar o los adquiridos a lo largo de crecimiento, para que éstos les permitan aprender a convivir y desenvolverse con otros seres humanos en la sociedad presente $\mathrm{y}$ futura, pero en contra parte la violencia que se aprecia actualmente en la sociedad de hoy en día está influyendo de manera muy profunda en la forma de percibir la vida por parte de los alumnos.

Conscientes de esta problemática, se han tomado también acciones en el ámbito del estado. El Plan Estatal de Desarrollo (20102016), en el apartado tres, enfocado a la formación para la vida y relacionado con el factor educativo, intenta fortalecer una formación con calidad al fomentar una educación integral proporcionando a los alumnos ambientes educativos propicios para el desarrollo de una visión crítica y reflexiva para que alumnos dentro de la educación básica puedan desarrollar sus capacidades intelectuales y reflexivas, para que aprendan a distinguir entre las alternativas que les presente la vida, la que pueda resolver sus problemas de la mejor manera y apegada a los valores universales (Gobierno del Estado de Chihuahua, 2010 ). Así mismo pretende lograr la participación de las comunidades escolares logrando espacios seguros y adecuados para el mejor desarrollo de los educandos sin perder de vista la construcción de una ciudadanía que se desarrolle dentro de los valores como la solidaridad, legalidad, respeto, equidad, igualdad y justicia.

Un clima escolar ideal provee las condiciones necesarias para que se geste de manera eficiente los procesos de enseñanza y aprendizaje, las relaciones personales que se dan dentro de un centro escolar y la forma en que conviven cotidianamente los seres humanos dentro del espacio educativo favorecen o entorpecen, según el caso, el desarrollo y desenvolvimiento normal del educando. La violencia y la agresividad que ésta conlleva limitan de manera importante el clima necesario para el éxito escolar académico y humano.

Los primeros estudios sobre el fenómeno se remontan a los años setenta en Europa, siendo después, durante los años ochenta cuando se realizan importantes aportaciones al tema en los países desarrollados. De los años noventa y hasta la fecha se han hecho investigaciones en los países de América Latina incluidas algunas efectuadas en México.

Así pues, en recientes fechas se ha avanzado en el reconocimiento del problema, terminando con la idea de que la escuela es un 
lugar totalmente seguro, aunque aún con un poco de resistencia por parte de ciertos representantes de las instituciones educativas. Algunas Instancias gubernamentales han hecho énfasis en la necesidad de implementar políticas públicas que respondan a las necesidades actuales de los estudiantes procurando que en las escuelas se enfatice la enseñanza de los principios universales de los derechos humanos en el ámbito escolar y a fomentar el humanismo en el currículo

\section{Metodología}

Hablando de educación, la finalidad y naturaleza de los fenómenos que se estudian indican el método propicio para acercarse al conocimiento de la realidad que se pretende mostrar. En el caso de la presente investigación, se trata de reconstruir las formas de pensar, sentir y actuar de los actores involucrados en el proceso de formación escolar. Hay indicadores observables o no que es posible interpretar al momento de entrar en contacto con lo que sucede dentro del contexto de la escuela; es necesario pues, utilizar procedimientos que nos permitan percibir y recuperar los significados en sus diferentes dimensiones, mediante un paradigma cualitativo.

Dado que el interés del estudio se centra en el conocimiento de las formas de concebir e interpretar la realidad, el método fenomenológico, asociado al filósofo Edmund Husserl se relaciona con todo lo que se percibe, ya sea de manera objetiva o subjetiva, en relación con una situación o aspecto a investigar. Este método rescata la forma de pensar de los investigados e intenta interpretar de una manera más objetiva un suceso.

En este método los investigadores realizan observaciones generales al principio y después intentan entender y develar el significado que las personas atribuyen la situación, para ello utilizan técnicas, como la entrevista, grupo focal, la observación o la encuesta. La expresión de las ideas de los participantes es de suma importancia, ya que permiten rescatar las percepciones de cada uno e interpretarlas con el fin de comprender el fenómeno que se estudia en la investigación.

En el caso específico de este trabajo, se emplearon técnicas de investigación como: la encuesta, la cual fue dirigida hacia los docentes y directivos, padres de familia y alumnos de cuarto, quinto y sexto grado de dos escuelas primarias ubicadas en la ciudad de Chihuahua. En esta etapa, los alumnos, docentes $y$ directivos tuvieron una participación muy destacada, mientras que los padres de familia mostraron cierta reserva, ya que una parte de ellos no regresó a la escuela la encuesta que se envió a la totalidad de ellos, a pesar de la insistencia por parte de los docentes. Con el uso de la encuesta, por medio de una serie de preguntas con diferentes opciones como respuesta se intenta conocer las percepciones de los adultos y los propios niños involucrados en el tema, la encuesta presenta al final de cada pregunta una opción abierta, a través de la cual, los participantes tuvieron la oportunidad de hacer sus comentarios acerca del tema, cabe resaltar que estos datos permitieron entender con mayor certeza las impresiones de los encuestados. También se utilizó la observación como técnica y el diario de campo como instrumento de registro, en el cual se registraron las observaciones realizadas dentro de los planteles, en este proceso se centró la atención en el ambiente escolar que se genera dentro de los dos contextos seleccionados. Se acudió a las escuelas a la hora de recreo, ya que este es el tiempo en el que los alumnos se sienten con mayor libertad y actúan de manera natural y sin restricciones. Se tuvo la oportunidad de presenciar la forma de actuar de los alumnos y de notar además la tendencia que tienen algunos de ellos a jugar 
integrando actitudes violentas dentro del juego. Aunado a las observaciones y la encuesta, se efectuaron entrevistas individuales semiestructuradas dirigidas a todos los actores involucrados, ofreciendo información personal acerca de la percepción individual de la problemática estudiada mediante la opinión de cada uno de ellos. Así mismo se llevaron a cabo dos entrevistas a grupo focal con alumnos de cuarto, quinto y sexto grado en cada una de las instituciones, donde se tuvo la oportunidad de conversar con niños que presentaban características similares: el primer grupo se enfocó a rescatar las opiniones de alumnos agresores o con tendencias violentas y que los docentes detectaron en sus respectivos grupos y en el segundo grupo se tuvo la participación de niños que han sido víctimas o están propensos a serlo. Esta técnica resultó sumamente interesante ya que así se tuvo la oportunidad de rescatar las diversas impresiones acerca de la misma problemática directamente de los actores principales de la misma. Los alumnos participaron favorablemente de manera sincera y abierta.

\section{Resultados}

Todos los instrumentos utilizados en las técnicas descritas anteriormente, evidencian que un gran número de estudiantes tiende a presentar conductas violentas a la hora de compartir el tiempo y el espacio en los centros escolares con sus similares.

La violencia entre compañeros resultó ser una situación cotidiana dentro de las instituciones educativas seleccionadas. Un gran porcentaje de los participantes, salvo algunas excepciones, coincidieron que la violencia entre pares sucede regularmente y que además es una situación que tiende a incrementarse. La mayoría está consciente de la gravedad del fenómeno y de las implicaciones que conlleva. Hay una manifestación generalizada en el sentido de la necesidad de buscar alternativas que

La violencia entre pares dentro del ambiente escolar permitan detener, como acción más urgente y posteriormente disminuir y prevenir este tipo de conductas.

Las encuestas aplicadas muestran datos interesantes acerca del fenómeno, entre los cuales se puede mencionar que existen diversas zonas dentro de los planteles escolares, que son propicias para el surgimiento de situaciones conflictivas, así como se coincidió en que durante todo el turno escolar pueden ocurrir hechos violentos. Una gran parte de los participantes están de acuerdo en que cuando se detecta a los alumnos agresivos o violentos, por lo regular se toman medidas correctivas, pero la eficacia de estás se encuentra en tela de juicio. También se encontró que generalmente a los niños que son víctimas de estas situaciones no tienen un seguimiento por parte de los responsables de su seguridad en cuanto a su situación se refiere. Se intentan resolver los conflictos pero no se continúa en el monitoreo de las conductas consecuentes, tanto de las víctimas como de los victimarios. En cuanto a la participación de los padres de familia con alumnos involucrados en situaciones de violencia entre pares, las opiniones se encuentran divididas, ya que algunos opinan que se toman acciones para participar en la solución de la problemática, mientras otros aseguran que hay un gran número de padres que no les importa la situación.

La violencia entre pares es un fenómeno que por mucho tiempo fue ignorado, a pesar de tener la certeza de su existencia, pero que con el incremento tanto en el número de participantes, como la diversificación de sus manifestaciones y su intensidad, ya no es posible mantenerse al margen, por lo que es necesario realizar este tipo de investigaciones y buscar la solución al problema.

La investigación se encuentra en proceso por lo que los resultados finales aún continúan en su definición. 


\section{Referencias}

Díaz-Aguado, M. (2005). Porqué se produce la violencia escolar y cómo prevenirla. Revista Iberoamericana de educación, (37), p. 19 y 22.

Furlán, A. (2010). Violencia en los centros educativos. México, D.F: Ediciones Novedades Educativas.

Gobierno del Estado de Chihuahua (2010). Plan Estatal de Desarrollo 2010-2016. Chihuahua, Chih.

Organización de Estados Iberoamericanos para la Educación, l. C. (2010). 2021 Metas Educativas. Madrid: Cudipal.
UNESCO. (2010).La crisis puede retrasar los progresos de la educación en el mundo. Recuperado el 28 de Enero de 2011, de http://www.oei.es/noticias/spip.php? article6403

Zorrilla, F. M. (2002). Diez años después del Acuerdo Nacional para la Modernización de la Educación Básica en México: Retos, tensiones y perspectivas. Recuperado el 16 de agosto de 2011, de http://redie.auabc.mx/vol4no2/impri mir-contenido- zorrilla.html 